\title{
LINEAR STABILITY OF THE HOPSCOTCH SCHEME
}

\author{
W.H. HUNDSDORFER and J.G. VERWER \\ Centre for Mathematics and Computer Science, P.O. Box 4079, 1009 AB Amsterdam, The Netherlands
}

\begin{abstract}
This paper is devoted to the hopscotch scheme, which is a numerical integration technique for time-dependent partial differential equations. We examine its linear stability properties. A general theorem is presented which provides sufficient conditions for boundedness of the numerical solution during time stepping on a fixed space-time mesh. This theorem has applications in the field of parabolic problems. For the one-space-dimensional convection-diffusion equation we present a detailed stability analysis of the odd-even scheme combined with central and one-sided finite differences. We compare stability based on the spectral condition with von Neumann stability.
\end{abstract}

Keywords: Partial differential equations, convection-diffusion equation, hopscotch method, linear stability.

\section{Introduction}

This paper is concerned with stability properties of the well-known hopscotch scheme, an efficient numerical integration method for time-dependent partial differential equations [2-6]. We shall examine the scheme for linear, homogeneous initial-boundary value problems. Particular attention will be given to the one-space-dimensional convection-diffusion equation.

Consider the linear differential system in $\mathbb{R}^{m}$ with constant coefficients

$$
\frac{\mathrm{d}}{\mathrm{d} t} U(t)=A U(t), \quad t \geqslant 0, \quad U(0)=U^{0}
$$

whereby it is supposed that (1.1) represents a semidiscrete, space-discretized, partial differential equation. Throughout the paper we let the positive parameter $h$ refer to the mesh width in space, which determines the dimension $m$.

Let $I_{1}$ and $I_{2}$ be two diagonal $m \times m$ matrices whose diagonal elements are either 0 or 1 , and such that $I_{1}+I_{2}=I$, the unit matrix. A hopscotch type scheme for (1.1) then fits into the formulation

$$
\begin{aligned}
& U^{n+1}=\left(I-\tau I_{2} A\right)^{-1}\left(I+\tau I_{1} A\right) U^{n}, \\
& U^{n+2}=\left(I-\tau I_{1} A\right)^{-1}\left(I+\tau I_{2} A\right) U^{n+1}
\end{aligned}
$$

(for $n$ even). Here $\tau>0$ is the stepsize in time and $U^{n}$ is the approximation to the exact solution $U$ of (1.1) at time $t_{n}=n \tau(n=1,2, \ldots)$. The choice of $I_{1}$ and $I_{2}$ determines the particular type of scheme (odd-even, line, etc.) [3-6]. With $A_{j}=\tau I_{j} A(j=1,2)$ we can write (1.2) as

$$
U^{n+2}=T U^{n}
$$

where

$$
T=\left(I-A_{1}\right)^{-1}\left(I+A_{2}\right)\left(I-A_{2}\right)^{-1}\left(I+A_{1}\right) .
$$

The conditions for stability of the scheme are governed by the matrix $T$. 
In Section 2 we consider the effect for large $n$ of an error in the initial value if $\tau$ and $h$ are fixed. Let $\|\cdot\|$ stand for a given norm on $\mathbb{R}^{m}$ as well as for the induced matrix norm. The hopscotch scheme (1.3) has a bounded error propagation iff

$$
\sup _{n \geqslant 0}\left\|T^{n}\right\| \leqslant \gamma
$$

for some $\gamma \in(0, \infty)$. It is well known that there is a close relation between inequality (1.5) and the spectral radius $\rho(T)$ of $T$. The spectral condition $\rho(T)<1$ is sufficient for (1.5) whereas $\rho(T) \leqslant 1$ is necessary. In Section 2.1 this will be made more precise, and we present sufficient conditions for (1.5) which are applicable for parabolic problems. Some related results of Gourlay [3] are generalized and improved. For the odd-even hopscotch scheme applied to the convection-diffusion equation, using either central or one-sided differences in space, we shall give in Section 2.3 conditions on $\tau$ and $h$ which are necessary and sufficient for (1.5).

The stability condition (1.5) is a rather weak one since the upper bound $\gamma$ is allowed to depend on the stepsizes $\tau$ and $h$. In particular for small $h$ this $\gamma$ may become intolerably large. For the forward Euler method applied to the convection-diffusion equation this phenomenon has been given much attention in the literature (see e.g. [1] and the references therein). We pay attention to this shortcoming of condition (1.5) in Section 3 of our paper.

\section{Stability for fixed grids}

\subsection{The spectral condition}

In order to formulate conditions equivalent to (1.5) we introduce some notation. By $L\left(\mathbb{R}^{m}\right), L\left(\mathbb{C}^{m}\right)$ we denote the space of $m \times m$ matrices which are real, complex, respectively. A matrix norm on $L\left(\mathbb{R}^{m}\right)$ induced by a given norm $\|\cdot\|$ on $\mathbb{R}^{m}$ is also denoted by $\|\cdot\|$. For a regular matrix $Q \in L\left(\mathbb{C}^{m}\right)$ we define the norm $\|\cdot\|_{Q}$ on $\mathbb{R}^{m}$ by $\|v\|_{Q}=\|Q v\|_{2}\left(\right.$ for $v \in \mathbb{R}^{m}$ ), where $\|\cdot\|_{2}$ stands for the Euclidean $\left(l_{2^{-}}\right)$norm. The induced matrix norm on $L\left(\mathbb{R}^{m}\right)$ is then given by $\|T\|_{Q}=\left\|Q T Q^{-1}\right\|_{2}$ (for $T \in L\left(\mathbb{R}^{m}\right)$ ). Following [13] we call an eigenvalue $\lambda$ of $T \in L\left(\mathbb{R}^{m}\right)$ defect if its (algebraic) multiplicity is $s$ whereas there do not exist $s$ linearly independent eigenvectors for this eigenvalue. Further $\sigma(T)$ will stand for the spectrum of $T$, and the spectral radius of $T$ is denoted by $\rho(T)$. For $R \in L\left(\mathbb{R}^{m}\right)$ or $L\left(\mathbb{C}^{m}\right)$ we write $R \geqslant 0$ if $\operatorname{Re}\left(v^{*} R v\right) \geqslant 0$ (for all $v \in \mathbb{C}^{m}$ ). Similarly we use the notations $R>0$ and $R \leqslant 0$. Note that symmetry of $R$ is not required. Finally, it is emphasized that throughout this section $\tau$ and $h$ (and thus $m$ ) are supposed to be fixed parameters.

Theorem 2.1. Let $\|\cdot\|$ stand for an arbitrary norm on $\mathbb{R}^{m}$. The following three statements are equivalent.

$$
\begin{aligned}
& \sup _{n \geqslant 0}\left\|T^{n}\right\|<\infty \\
& \rho(T) \leqslant 1 \text { and any } \lambda \in \sigma(T) \text { with }|\lambda|=1 \text { is not defect, } \\
& \text { there exists a regular } Q \in L\left(\mathbb{C}^{m}\right) \text { such that }\|T\|_{Q} \leqslant 1 .
\end{aligned}
$$


Proof. By writing $T$ in its Jordan canonical form, it follows from [16, Theorem 3.1] that $\rho(T) \leqslant 1$ iff there is a $\gamma>0$ such that $\left\|T^{n}\right\| \leqslant 1+\gamma n^{q-1}(n=0,1,2, \ldots)$ where $q$ is the maximal order of the Jordan blocks belonging to an eigenvalue $\lambda$ of $T$ with $|\lambda|=1$. We see that (2.1) holds iff $q=1$, which shows the equivalence between (2.1) and (2.2).

The equivalence between (2.2) and (2.3) follows from the material presented in [9, Section 2.3] (cf. also [13]).

Condition (2.2), a spectral condition, will be employed later for finding necessary and sufficient conditions for (2.1) for a concrete problem, the convection-diffusion equation. First we shall derive sufficient conditions for general problems by using the equivalence between (2.1) and (2.3).

It is clear that (2.1)-(2.3) hold for $T$ iff these statements hold for a matrix $S \in L\left(\mathbb{R}^{m}\right)$ which is similar to $T$. We define

$$
\begin{aligned}
& S_{j}=\left(I-A_{j}\right)^{-1}\left(I+A_{j}\right), \quad j=1,2, \\
& S=S_{2} S_{1} .
\end{aligned}
$$

We then have the similarity

$$
T=\left(I-A_{1}\right)^{-1} S\left(I-A_{1}\right) .
$$

Further it is clear that $\left\|S_{j}\right\|_{Q} \leqslant 1(j=1,2)$ is sufficient for $\|S\|_{Q} \leqslant 1$.

Lemma 2.2. Suppose $Q \in L\left(\mathbb{C}^{m}\right)$ is regular, and let $R=Q^{*} Q$. We have $\left\|S_{j}\right\|_{Q} \leqslant 1(j=1,2)$ iff $B=A R^{-1}$ satisfies $B \leqslant 0, I_{1} B=B I_{1}$.

Proof. From [11, Lemma 2 and its proof] it follows that $\left\|S_{j}\right\|_{Q} \leqslant 1(j=1,2)$ iff $Q A_{j} Q^{-1} \leqslant 0(j$ $=1,2$ ). It is easily seen that the latter inequalities are equivalent to

$$
I_{j} B \leqslant 0, \quad j=1,2 .
$$

The conditions $B \leqslant 0, I_{1} B=B I_{1}$ are sufficient for (2.6) because $B \leqslant 0$ implies $I_{j} B I_{j} \leqslant 0(j=$ $1,2)$, and from $I_{1} B=B I_{1}$ it follows that $I_{j} B=I_{j} B I_{j}(j=1,2)$.

On the other hand, since $B=I_{1} B+I_{2} B$, relation (2.6) implies $B \leqslant 0$. In order to show that (2.6) also implies $I_{1} B=B I_{1}$ we put

$$
V_{j}=\left\{w \in \mathbb{C}^{m}: I_{j} w=w\right\}, \quad j=1,2 .
$$

Any $v \in \mathbb{C}^{m}$ can be written as $v_{1}+v_{2}$ with $v_{j}=I_{j} v \in V_{j}$, and then

$$
v^{\mathrm{T}} I_{j} B v=v_{j}^{\mathrm{T}} I_{j} B v_{j}+v_{j}^{\mathrm{T}} I_{j} B v_{k}, \quad j=1,2, \quad k=3-j .
$$

Using (2.6) it follows that $v_{j}^{\mathrm{T}} I_{j} B v_{k}=0(j=1,2$ and $k=3-j)$ for arbitrary $v_{1} \in V_{1}, v_{2} \in V_{2}$, and since we can choose $v_{j}=I_{j} B v_{k}$ we obtain $I_{j} B I_{k} v=0$ (for all $v \in \mathbb{C}^{m}, j=1,2$ and $k=3-j$ ). The relation $I_{1} B=B I_{1}$ now easily follows.

Theorem 2.3. Suppose $A=B R$ for some $B, R \in L\left(\mathbb{C}^{m}\right)$ with $R^{*}=R>0$ and $B \leqslant 0, I_{1} B=B I_{1}$. Then the hopscotch scheme is stable in the sense of (2.1) (for fixed $\tau$ and $h$ ). 
The proof of this theorem directly follows by combining Theorem 2.1 and Lemma 2.2. If

$$
M=\left\{j: j \in \mathbb{N}, 1 \leqslant j \leqslant m, j \text { th diagonal element of } I_{1} \text { equals } 1\right\},
$$

then $I_{1} B=B I_{1}$ iff the entries $b_{i j}$ of $B$ satisfy $b_{i j}=0$ for $i \in M, j \notin M$ or $j \in M, i \notin M$. Fo instance for the odd-even hopscotch scheme, where $M$ is the set of even integers between 1 anc $m$, the condition on $B$ reads $b_{i j}=0$ if $i-j=1(\bmod 2)$.

Applications of Theorem 2.3 lie in the field of parabolic equations as expressed in thi following corollary. We note that a closely related, slightly less general result can be proved b: using a corollary of $[15, \mathrm{p} .184]$.

Corollary 2.4. Suppose $A=-D R$ with $D \in L\left(\mathbb{R}^{m}\right)$ diagonal with nonnegative entries and $R \in$ $L\left(\mathbb{R}^{m}\right)$ symmetric and positive-definite. Then (2.1) holds.

Remark 2.5. Corollary 2.4 is a generalization of Gourlay [3, Theorem 1]. His theorem is obtained if we set $D=I$. Theorem 2 in the same paper is obtained if we set $R=I$ in our Theorem 2.3 However the matrix $A$ then must satisfy $I_{1} A=A I_{1}$ which is a severe and unnatural conditior (see above and also [15, Remark 2.5, p. 183]). Finally we note that the statements of [3, Lemm 3(i)] and [3, Theorems 3 and 4] are not correct. For any of these statements it is possible tc construct a simple counterexample with a $2 \times 2$ matrix $A$ (= $=-H$ in the notation of [3]). For instance, the diagonally dominant matrix

$$
A=a\left(\begin{array}{cc}
-1 & 1 \\
-\frac{1}{2} & -1
\end{array}\right)
$$

with $a>0$ sufficiently large, contradicts the statements of [3, Theorems 3 and 4].

\subsection{Permuted forms}

In the previous section we have derived sufficient conditions for the stability inequality by using (2.3). In the following we employ (2.2) to derive necessary and sufficient conditions ir some cases. We then have to deal directly with $S$, or with $T$, instead of $S_{1}$ and $S_{2}$. To make this feasible we introduce the permutation matrix $P \in L\left(\mathbb{R}^{m}\right)$ such that

$$
P I_{1} P^{\mathrm{T}}=\left(\begin{array}{ll}
I & 0 \\
0 & 0
\end{array}\right), \quad P I_{2} P^{\mathrm{T}}=\left(\begin{array}{ll}
0 & 0 \\
0 & I
\end{array}\right)
$$

and we denote

$$
A^{\prime}=P A P^{\mathrm{T}}=\left(\begin{array}{ll}
A_{11} & A_{12} \\
A_{21} & A_{22}
\end{array}\right) .
$$

For given $I_{1}$ and $I_{2}$ we shall specify later on the matrix $P$.

Consider the matrix $S$ defined by (2.5), and let $S^{\prime}=P S P^{\mathrm{T}}$. By some calculations we obtair.

$$
S^{\prime}=\left(\begin{array}{ll}
S_{11} & S_{12} \\
S_{21} & S_{22}
\end{array}\right)
$$


with

$$
\begin{aligned}
& S_{11}=\left(I-A_{11}\right)^{-1}\left(I+A_{11}\right), \quad S_{12}=2\left(I-A_{11}\right)^{-1} A_{12}, \\
& S_{21}=2\left(I-A_{22}\right)^{-1} A_{21}\left(I-A_{11}\right)^{-1}\left(I+A_{11}\right), \\
& S_{22}=\left(I-A_{22}\right)^{-1}\left(I+A_{22}\right)+4\left(I-A_{22}\right)^{-1} A_{21}\left(I-A_{11}\right)^{-1} A_{12} .
\end{aligned}
$$

This form, although still rather complicated, enables us to calculate the spectral radius of $S$, and thus of $T$, in some simple situations.

As an application we shall consider the odd-even hopscotch scheme applied to the convection-diffusion equation where the convection term is discretized by central or one-sided differences. One can show that for the matrix $A$ which then arises (see (2.11)) Theorem 2.3 is not applicable. On the other hand the sufficient conditions of Theorem 2.3 and Corollary 2.4, which are useful for parabolic problems, do not easily follow from the permuted forms.

\subsection{An application of the permuted forms}

In this section we consider the odd-even hopscotch scheme. Suppose for convenience that $m$ is even and set $m^{\prime}=\frac{1}{2} m$. Let $I_{1}=\operatorname{diag}(1,0,1,0, \ldots, 1,0)$ and let the permutation matrix $P$ defined by

$$
[P v]_{i}=v_{2 i-1}, \quad[P v]_{m^{\prime}+i}=v_{2 i} \quad \text { for } 1 \leqslant i \leqslant m^{\prime}, \quad v \in \mathbb{R}^{m} .
$$

Thus $P$ puts all the odd components in the first $m^{\prime}$ positions and the even ones in the last positions.

We shall analyze the stability properties of this scheme for the convection-diffusion problem:

$$
\begin{aligned}
& \frac{\partial}{\partial t} u(x, t)+a \frac{\partial}{\partial x} u(x, t)=b \frac{\partial^{2}}{\partial x^{2}} u(x, t), \quad t>0, \quad 0<x<1, \\
& u(x, 0) \text { given, } \quad 0 \leqslant x \leqslant 1, \quad u(0, t)=u(1, t)=0, \quad t \geqslant 0,
\end{aligned}
$$

where $a>0$ and $b>0$ are given parameters. For the space discretization we choose a uniform grid with mesh width $h=1 /(m+1)$. For the diffusion term $b u_{x x}$ we use standard second-order differences. The convection term $a u_{x}$ is discretized either by central or by one-sided (upwind) differences.

Let $E$ stand for the shift operator in $\mathbb{R}^{m}$ or $\mathbb{R}^{m^{\prime}}$,

$$
E=\left(\begin{array}{ccccc}
0 & 1 & & & \\
& 0 & 1 & & \\
& & \ddots & \ddots & \\
& & & & 1 \\
& & & & 0
\end{array}\right)
$$

The matrix $A$ arising in the semidiscrete approximation (1.1) to problem (2.10) is given by

$$
A= \begin{cases}-\frac{a}{2 h}\left(-E^{\mathrm{T}}+E\right)+\frac{b}{h^{2}}\left(E^{\mathrm{T}}-2 I+E\right) & \text { for central differences, } \\ -\frac{a}{h}\left(-E^{\mathrm{T}}+I\right)+\frac{b}{h^{2}}\left(E^{\mathrm{T}}-2 I+E\right) & \text { for one-sided differences. }\end{cases}
$$


In both cases we write

$$
\tau A=-\alpha\left(-E^{\mathrm{T}}+E\right)+\beta\left(E^{\mathrm{T}}-2 I+E\right),
$$

where $\alpha=\frac{1}{2} \tau a / h, \beta=\tau b / h^{2}$ in case of central differences, and $\alpha=\frac{1}{2} \tau a / h, \beta=\frac{1}{2} \tau a / h+\tau b / h^{2}$ for one-sided differences. We define $V, W \in L\left(\mathbb{R}^{m^{\prime}}\right)$ by

$$
V=\beta\left(E^{\mathrm{T}}+I\right), \quad W=\alpha\left(E^{\mathrm{T}}-I\right) .
$$

For the permuted forms of $\tau A$ and $S$ we obtain:

$$
\begin{aligned}
\tau A^{\prime} & =\left(\begin{array}{cc}
-2 \beta I & V+W \\
V^{\mathrm{T}}-W^{\mathrm{T}} & -2 \beta I
\end{array}\right), \\
S^{\prime} & =\left(\begin{array}{cc}
\frac{1-2 \beta}{1+2 \beta} I & \frac{2}{1+2 \beta}(V+W) \\
2 \frac{1-2 \beta}{(1+2 \beta)^{2}}\left(V^{\mathrm{T}}-W^{\mathrm{T}}\right) & \frac{1-2 \beta}{1+2 \beta} I+\frac{4}{(1+2 \beta)^{2}}\left(V^{\mathrm{T}}-W^{\mathrm{T}}\right)(V+W)
\end{array}\right) .
\end{aligned}
$$

Lemma 2.6. Let

$$
M=4(1+2 \beta)^{-2}\left(V^{\mathrm{T}}-W^{\mathrm{T}}\right)(V+W) \quad \text { and } \quad \nu=(1-2 \beta) /(1+2 \beta) .
$$

We have $\lambda \in \sigma(T)$ iff $\lambda^{2}-(\mu+2 \nu) \lambda+\nu^{2}=0$ for some $\mu \in \sigma(M)$.

Proof. Let $\tilde{\lambda}=\lambda-\nu$ for $\lambda \in \sigma(T)=\sigma\left(S^{\prime}\right)$. We have

iff

$$
S^{\prime} v=\lambda v, \quad v=\left(v_{1}^{\mathrm{T}}, v_{2}^{\mathrm{T}}\right)^{\mathrm{T}} \neq 0
$$

$$
\begin{aligned}
& \tilde{\lambda} v_{1}=\frac{2}{1+2 \beta}(V+W) v_{2}, \\
& \tilde{\lambda} v_{2}=2 \frac{(1-2 \beta)}{(1+2 \beta)^{2}}\left(V^{\mathrm{T}}-W^{\mathrm{T}}\right) v_{1}+\frac{4}{(1+2 \beta)^{2}}\left(V^{\mathrm{T}}-W^{\mathrm{T}}\right)(V+W) v_{2} .
\end{aligned}
$$

By considering the cases $\tilde{\lambda}=0$ and $\tilde{\lambda} \neq 0$ separately, it follows by some calculations that such $v_{1}$ and $v_{2}$ exist iff the matrix $\tilde{\lambda}^{2} I-(\tilde{\lambda}+\nu) M$ is singular, which is equivalent to the statement

$$
\tilde{\lambda}^{2}-(\tilde{\lambda}+\nu) \mu=0 \text { for some } \mu \in \sigma(M) .
$$

Substitution of $\tilde{\lambda}=\lambda-\nu$ completes the proof.

In order to use this lemma we investigate the spectrum of $M$. We have

$$
M=\frac{4}{(1+2 \beta)^{2}}\left[(\alpha+\beta)^{2} E^{\mathrm{T}}+\left(\beta^{2}-\alpha^{2}\right)\left(I+E E^{\mathrm{T}}\right)+(\alpha-\beta)^{2} E\right] .
$$

If $\alpha=\beta$ the spectrum of $M$ only consists of 0 . If $\alpha \neq \beta$, then

$$
M=4 \frac{\left(\beta^{2}-\alpha^{2}\right)}{(1+2 \beta)^{2}} N\left(\frac{\beta-\alpha}{\beta+\alpha}\right),
$$


where

$$
N(\xi)=\xi^{-1} E^{\mathrm{T}}+\left(I+E E^{\mathrm{T}}\right)+\xi E \quad \text { for } \xi \in \mathbb{R}, \quad \xi \neq 0
$$

$\mathrm{s}$ an $m^{\prime} \times m^{\prime}$ matrix. We have the similarity

$$
N(\xi)=\operatorname{diag}\left(\xi^{m^{\prime}}, \xi^{m^{\prime}-1}, \ldots, \xi\right) N(1) \operatorname{diag}\left(\xi^{m^{\prime}}, \xi^{m^{\prime}-1}, \ldots, \xi\right)^{-1}
$$

ind the eigenvalues of $N(1)$ are given by $2+2 \cos \left(2 j \pi /\left(2 m^{\prime}+1\right)\right)\left(1 \leqslant j \leqslant m^{\prime}\right)$ (see e.g. [7, Example 7.5]). Consequently the spectrum of $M$ is given by $\left\{\mu_{1}, \mu_{2}, \ldots, \mu_{m^{\prime}}\right\}$ where

$$
\mu_{j}=4 \frac{\left(\beta^{2}-\alpha^{2}\right)}{(1+2 \beta)^{2}}[2+2 \cos (2 j \pi h)], \quad 1 \leqslant j \leqslant m^{\prime} .
$$

Note that this also holds if $\alpha=\beta$.

To formulate the main result of this section we consider the following condition

$$
\alpha^{2} \leqslant \frac{1}{4}+\beta^{2} \text {. }
$$

Theorem 2.7. Let $\alpha \geqslant 0, \beta>0$. Condition (2.16) implies $\rho(T)<1$. On the other hand if $(2.16)$ does not hold we have $\rho(T)>1$ for $h>0$ sufficiently small.

Proof. Let $<$ stand consistently for either $\leqslant$ or $<$. According to Lemma 2.6 the eigenvalues $\lambda$ of $T$ are given by

$$
\lambda^{2}-(\mu+2 \nu) \lambda+v=0,
$$

where $\mu$ ranges over $\sigma(M)$. The roots $\lambda$ of this equation have modulus $<1$ iff $|\nu|<1$ and $|\mu+2 \nu|<1+\nu^{2}$. For any $\beta>0$ we have $|\nu|<1$. It follows that $\rho(T)<1$ iff for all $\mu \dot{\in} \sigma(M)$ there holds

$$
\left(\mu-(1-\nu)^{2}\right)\left(\mu+(1+\nu)^{2}\right)<0
$$

i.e.

$$
-(1+\nu)^{2}<\mu<(1-\nu)^{2}
$$

Using (2.15) we see that $\rho(T)<1$ iff

$$
-1<\left(\beta^{2}-\alpha^{2}\right)(1+\cos (2 j \pi h))<4 \beta^{2} \text { for } j=1,2, \ldots, m^{\prime} .
$$

From this relation the proof now easily follows.

For central differences condition (2.16) can be written as

$$
\tau^{2}\left(a^{2}-4 b^{2} / h^{2}\right) \leqslant h^{2}
$$

Hence, if $a \leqslant 2 b / h$ there is no restriction on $\tau$ for having stability in the sense of (2.1). In case we deal with one-sided differences condition (2.16) always holds, and thus $\rho(T)<1$ for any ratio of $\tau$ and $h$. We note that with one-sided differences this stability result is also valid if $b=0$ (no diffusion). Of course, for the problem (2.10) to be well posed we then should omit the boundary condition $u(1, t)=0$. 


\section{Stability uniformly in the mesh width}

As observed previously the spectral condition and the related inequality (1.5) are not very reliable for ensuring stability in a practical situation, and not sufficient to establish convergence. This fact is well known, especially for the forward Euler method applied to the convection diffusion problem. Here we discuss it for the hopscotch scheme.

If $\|\cdot\|$ stands for a prescribed norm on $\mathbb{R}^{m}$ for $(m \in \mathbb{N})$ the stability constant $\gamma$ in (1.5) may tend to infinity if $h \downarrow 0$ (even if at the same time $\tau \downarrow 0$; see e.g. [1, Section 10.5; 12]). Instead of stability for fixed grids it is preferable to have

$$
\left\|T^{n}\right\|_{p} \leqslant \gamma \quad \text { for all } n \in \mathbb{N}, \quad h>0, \quad \tau \leqslant \psi(h),
$$

say for some $l_{p}$ norm, with $\psi$ a positive function on $(0, \infty)$. This is sufficient for convergence (provided we have consistency), due to the fact that we then have stability uniformly in $h$ and $m$. Sufficient for (3.1) is $l_{p}$ contractivity

$$
\|T\|_{p} \leqslant 1 \quad \text { for all } h>0, \quad \tau \leqslant \psi(h) .
$$

This definitively excludes any error growth (in $l_{p}$ ). Moreover (3.2) is a natural condition in case the solutions $U$ of (1.1) are such that

$$
\|U(t+\tau)\|_{p} \leqslant\|U(t)\|_{p} \quad \text { for all } t \geqslant 0, \quad \tau>0
$$

for any $h>0$ and starting vector $U^{0} \in \mathbb{R}^{m}$. This happens to be so for the semidiscrete version of problem (2.10) with $p=2$ in case of central differences and with $p=1,2, \infty$ in case of one-sided differences (see e.g. [1, Section 10.6]).

Unfortunately for the hopscotch scheme such desirable stability inequalities (3.2) are very hard to analyze, even in the relatively simple case of the odd-even hopscotch scheme applied to problem (2.10). By using the permuted form $T^{\prime}=P T P^{\mathrm{T}}$ one can show that in case we are dealing with one-sided differences and $b=0$ (omitting the right boundary condition) we have $\|T\|_{\infty} \leqslant 1$ iff $\tau a \leqslant h$, whereas $\|T\|_{1}>1$ for any ratio of $\tau$ and $h$. If $b>0$ the situation already becomes very complicated.

For many well-known schemes applied to pure initial value problems with constant coefficients $l_{2}$ contractivity can be investigated by using Fourier transformations (von Neumann analysis). This analysis cannot be applied directly to the hopscotch scheme due to the fact that Fourier modes are not eigenvectors of $T$. However, by discharging half of the grid points, von Neumann analysis is applicable. For instance consider again the convection-diffusion equation (2.10). Suppose that $A$ is defined as in Section 2.3, and that

$$
\left[I_{1} U^{n}\right]_{j}=\left\{\begin{array}{cc}
U_{j}^{n} & \text { for } j+n \text { odd } \\
0 & \text { for } j+n \text { even }
\end{array}\right.
$$

(the odd-even partitioning). Then, if we ignore boundaries, the hopscotch approximations with $j+n$ odd satisfy the two-step scheme

$$
U_{j}^{n+2}=U_{j}^{n}-2 \tilde{\alpha}\left(U_{j+1}^{n+1}-U_{j-1}^{n+1}\right)+2 \tilde{\beta}\left(U_{j+1}^{n+1}+U_{j-1}^{n+1}-U_{j}^{n+2}-U_{j}^{n}\right)
$$

in case of central differences, and

$$
U_{j}^{n+2}=U_{j}^{n}-2 \tilde{\alpha}\left(U_{j}^{n}+U_{j}^{n+2}-2 U_{j-1}^{n+1}\right)+2 \tilde{\beta}\left(U_{j+1}^{n+1}+U_{j-1}^{n+1}-U_{j}^{n+2}-U_{j}^{n}\right)
$$



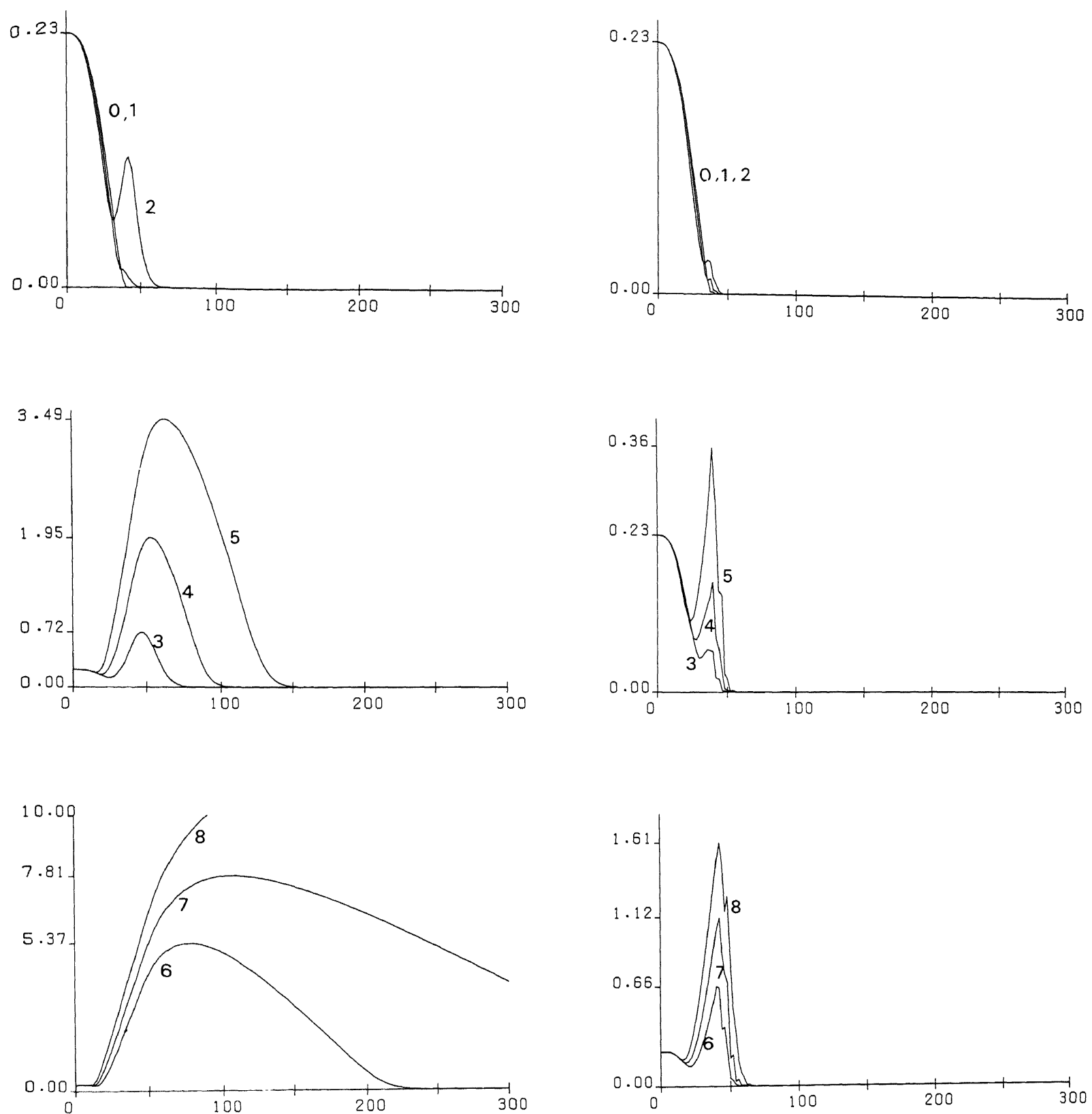

Fig. 1. The plots on the left correspond to central differences and the plots on the right to one-sided differences.

for one-sided differences. Here $\tilde{\alpha}=\frac{1}{2} a \tau / h, \tilde{\beta}=b \tau / h^{2}$. We refer to $[6,14]$ for details on the derivations. Both schemes live on the uncoupled set of odd points. They are of the Du Fort-Frankel type if $\tilde{\boldsymbol{\beta}}>0$, and if $\tilde{\boldsymbol{\beta}}=0$ (3.3) reduces to the leapfrog method. Fourier analysis is applicable now to these schemes in a standard way. It can be shown $[6,14]$ that both schemes are stable in the strict sense of von Neumann iff

$$
\tilde{\boldsymbol{\alpha}}^{2} \leqslant \frac{1}{4},
$$


i.e. $\tau a \leqslant h$. Stability in the strict sense of von Neumann is here taken to mean for any Fourier mode $U_{j}^{n}=\xi^{n} \mathrm{e}^{i \omega h j}$ we demand $|\xi| \leqslant 1$ for all $\omega h \in \mathbb{R}$.

Comparing (3.5) with the results of Section 2.3 (inequality (2.16)) we see that the spectral condition $\rho(T)<1$ permits a larger time step. To illustrate that results based on the spectral condition may be too optimistic we have carried out the following numerical experiment.

Using the problem parameters $a=1, b=0.01$ and the initial function $u(x, 0)=\sin \pi x$, we have applied the two schemes (3.3) and (3.4) on a fixed space grid with $h=\frac{1}{40}$ for niné values of $\tau$ given by $\tau^{-1}=40-2 i(0 \leqslant i \leqslant 8)$. Only for $\tau=\frac{1}{40}$ condition (3.5) is satisfied while for the larger stepsizes the spectral condition still holds (the critical value for (2.17) is $\tau=\frac{1}{24}$ ). For all integrations we have plotted $\log _{10}\left(1+\left\|U^{2 n}\right\|_{2}\right)$ as a function of $n$ (see Fig. 1). The integers $0,1, \ldots, 8$ near the graphs correspond to $\tau$ according to the relation $\tau^{-1}=40-2 i(0 \leqslant i \leqslant 8)$.

The growth of the solutions for large $\tau$ is clearly visible. In particular it is very strong for the scheme with central differences if $\tau$ approaches $\frac{1}{24}$. From a practical point of view this scheme can then no longer be called stable. On the other hand, for the one-sided scheme, which can be viewed as a central scheme with a larger (artificial) viscosity, the growth remains reasonably small for all the stepsizes. Thus for this example viscosity clearly helps for getting more stability, a fact which is not revealed by the von Neumann condition (3.5) (where boundaries are ignored).

Finally we note that the initial decay of the solutions, which happens for all the stepsizes, is due to our very smooth initial function. With a less smooth $u(x, 0)$ the same pictures arise but without this decay.

In conclusion one can say that although the spectral condition gives no guarantee for stability (uniform in $h$ ) this condition does provide some useful information. It is a necessary condition for stronger stability inequalities such as (3.1) and (3.2). In cases where such stronger stability inequalities are very hard to analyze (nonconstant coefficients, boundaries) the spectral condition still may be relatively easy to check (e.g. as in Section 2.1), giving us some idea on permissible stepsizes.

\section{Acknowledgment}

The authors are grateful to Joke Blom for carrying out the numerical experiments.

\section{References}

[1] K. Dekker and J.G. Verwer, Stability of Runge-Kutta methods for stiff nonlinear differential equations (North-Holland, Amsterdam, 1984).

[2] P. Gordon, Nonsymmetric difference equations, SIAM J. Appl. Math. 13 (1965) 667-673.

[3] A.R. Gourlay, Hopscotch: A fast second order partial differential equation solver, J. Inst. Math. Appl. 6 (1970) 375-390.

[4] A.R. Gourlay, Some recent methods for the numerical solution of time-dependent partial differential equations, Proc. Roy. Soc. London A. 232 (1971) 219-235.

[5] A.R. Gourlay, Splitting methods for time dependent partial differential equations, in: D. Jacobs, ed., The State of the Art in Numerical Analysis (Academic Press, New York, 1977) 757-791.

[6] A.R. Gourlay and J.LI. Morris, Hopscotch difference methods for nonlinear hyperbolic systems, IBM J. Res. Develop. 16 (1972) 349-353. 
[7] R.T. Gregory and D.L. Karney, A Collection of Matrices for Testing Computational Algorithms (Wiley-Interscience, New York, 1969).

[8] A.C. Hindmarsh, P.M. Gresho and D.F. Griffiths, The stability of explicit Euler time-integration for certain finite-difference approximations of the multi-dimensional advection-diffusion equation, Internat. J. Numer. Methods Fluids 4 (1984) 853-897.

[9] A.S. Householder, The Theory of Matrices in Numerical Analysis (Blaisdell, New York, 1964).

[10] J.J.H. Miller, On the location of zeros of certain classes of polynomials with applications to numerical analysis, $J$. Inst. Math. Appl. 8 (1971) 397-406.

[11] R.B. Kellogg, An alternating direction method for operator equations, J. SIAM 12 (1964) 848-853.

[12] K.W. Morton, Stability of finite difference approximations to a diffusion-convection equation, Internat. J. Numer. Math. Engrg. 15 (1980) 677-683.

[13] T. Ström, On logarithmic norms, SIAM J. Numer. Anal. 12 (1975) 741-753.

[14] J.H.M. ten Thije Boonkkamp and J.G. Verwer, On the odd-even hopscotch scheme for the numerical integration of time-dependent partial differential equations, Appl. Numer. Math. 3 (1987) 183-193.

[15] E.J.W. ter Maten, Stability analysis of finite difference methods for fourth order parabolic differential equations, Thesis, University of Utrecht, Netherlands (1984).

[16] R.S. Varga, Matrix Iterative Analysis (Prentice-Hall, Englewood Cliffs, NJ, 1962). 